\title{
Fuzzy soft set approach to ideal theory of regular AG-groupoids
}

\author{
Feng Feng, Madad Khan, Violeta Leoreanu-Fotea, \\ Saima Anis and Nadeem Ajaib
}

\begin{abstract}
This paper aims to apply fuzzy sets and soft sets in combination to investigate algebraic properties of regular AG-groupoids. We initiate $\left(\in_{\gamma}, \in_{\gamma} \vee q_{\delta}\right)$-fuzzy soft left ideals (right ideals, bi-ideals and quasiideals) over AG-groupoids and explore some related properties. Moreover, we give a number of characterizations for regular AG-groupoids by virtue of various types of fuzzy soft ideals over them.
\end{abstract}

\section{Introduction}

Uncertainty plays an essential role in the real world. In various fields such as data analysis, pattern recognition, expert systems and intelligent decision making, it has been found that mathematical models based on classical sets might not be suitable for dealing with problems containing vagueness and uncertainty. Also it is worth noting that uncertainties emerging from miscellaneous domains are very distinct and cannot be captured within a unique framework. In response to this, a number of useful mathematical theories such as probability theory, fuzzy sets [1], rough sets [2] and interval mathematics [3] have been developed.

Molodtsov's soft set theory [4] is a relatively new mathematical model for coping with uncertainty from a parametrization point of view. In mathematical modelling, sometimes it is impossible to find an exact solution of

Key Words: Groupoid, AG-groupoid, Soft set, Fuzzy set, Ideal

2010 Mathematics Subject Classification: Primary 20N02, 03E72; Secondary 06D72.

Received: December 20, 2014.

Accepted: January 7, 2015. 
the model of extremely high complexity. Alternatively, we are interested in finding numerical or approximate solutions. But this process can be done in a reverse way, that is, we can model and manipulate many real-life problems in an approximate way. By means of parameterization, a soft set can produce a series of approximate descriptions for a complicated entity being perceived from different aspects. This is in accordance with the way that humans use to treat uncertain concepts in their daily life. In recent years, soft set theory has been applied to various kinds of algebraic structures $[5,6,7,8,9,10,11,12,13,14,17,15,16,18,19,20,21]$. But so far as we know, the algebras which have been investigated in virtue of soft sets are all associative, and very little attention has been paid to non-associative algebraic structures in the literature.

An Abel-Grassmann's groupoid (or briefly, AG-groupoid) is a nonassociative algebraic structure lies in between a groupoid and a commutative semigroup. If an AG-groupoid contains left identity then this left identity is unique. An AG-groupoid with right identity becomes a commutative semigroup with identity. Moreover, every commutative AG-groupoid becomes a commutative semigroup. In most cases, an AG-groupoid behaves like a commutative semigroup. For instance, $a^{2} b^{2}=b^{2} a^{2}$ holds in a commutative semigroup, while this equation also holds for an AG-groupoid with left identity.

The aim of this study is to investigate ideal theory of regular AG-groupoids in virtue of fuzzy soft sets. In particular, we will introduce various types of $\left(\in_{\gamma}, \in_{\gamma} \vee q_{\delta}\right)$-fuzzy soft ideals over AG-groupoids and try to obtain some characterizations for regular AG-groupoids based on algebraic ideal theory of them.

\section{AG-Groupoids}

Let $(S,$.$) be a groupoid and a, b, c, d \in S$. It is said that $S$ is an AG-groupoid, if it satisfies the left invertive law $(a b) c=(c b) a$. It can be verified that the medial law $(a b)(c d)=(a c)(b d)$ holds in every AG-groupoid. If $S$ is an AG-groupoid with left identity $e$, then $S=S^{2}$ and the paramedial law $(a b)(c d)=(d b)(c a)$ holds. Also we have

$$
a(b c)=b(a c) .
$$

Moreover, it is easy to see that every one sided ideal of $S$ is a quasi-ideal of $S$. In [22], it shown that $L[a]=a \cup S a$ is a principal left ideal of $S$ generated by $a$. Note also that $B[a]=a \cup a^{2} \cup(a S) a$ is a principal bi-ideal and $Q[a]=a \cup(S a \cap a S)$ is a principle quasi-ideal of $S$ generated by $a$. A subset $B$ of $S$ is called generalized bi-ideal of $S$ if $(B S) B \subseteq B$. 
Lemma 1. Let $S$ be an AG-groupoid. If $a=a(a x)$ for some $x \in S$, then there exists $y \in S$ such that $a=a^{2} y$.

Proof. Assume that $a=a(a x)$ for some $x$ in $S$. By the medial law, we get

$$
a=a(a x)=[a(a x)](a x)=(a a)((a x) x)=a^{2} y, \text { where } y=(a x) x .
$$

Lemma 2. Let $S$ be an AG-groupoid with left identity. If $a=a^{2} x$ for some $x \in S$, then $a=($ ay $)$ a for some $y \in S$.

Proof. Suppose that $a=a^{2} x$ for some $x \in S$. Note first that

$$
a=a^{2} x=(a a) x=\left(\left(a^{2} x\right)\left(a^{2} x\right)\right) x .
$$

Then by the left invertive law, and the medial law, it follows that

$$
\left(\left(a^{2} x\right)\left(a^{2} x\right)\right) x=\left(\left(a^{2} a^{2}\right)(x x)\right) x=\left(x x^{2}\right)\left(a^{2} a^{2}\right) .
$$

According to Eq. (1), we get

$$
\left(x x^{2}\right)\left(a^{2} a^{2}\right)=a^{2}\left(\left(x x^{2}\right) a^{2}\right)=(a a)\left(\left(x x^{2}\right) a^{2}\right),
$$

and hence

$$
(a a)\left(\left(x x^{2}\right) a^{2}\right)=\left(\left(\left(x x^{2}\right) a^{2}\right) a\right) a=\left(\left(a a^{2}\right)\left(x x^{2}\right)\right) a,
$$

by the left invertive law. But we also have

$$
\left(a a^{2}\right)\left(x x^{2}\right)=\left(x^{2} a^{2}\right)(x a)=\left(x^{2} x\right)\left(a^{2} a\right),
$$

by both the paramedial law and the medial law. This implies that

$$
\left(\left(a a^{2}\right)\left(x x^{2}\right)\right) a=\left(\left(x^{2} x\right)\left(a^{2} a\right)\right) a .
$$

Using Eq. (1), the paramedial law and the medial law, we further deduce that

$$
\begin{aligned}
\left(\left(x^{2} x\right)\left(a^{2} a\right)\right) a & =\left(a^{2}\left(\left(x^{2} x\right) a\right)\right) a=\left((a a)\left(\left(x^{2} x\right) a\right)\right) a \\
& =\left(\left(a\left(x^{2} x\right)\right)(a a)\right) a=\left(a\left(\left(\left(a\left(x^{2} x\right)\right) a\right)\right) a=(a y) a,\right.
\end{aligned}
$$

where $y=\left(a\left(x^{2} x\right)\right) a \in S$. 
Lemma 3. Let $S$ be an AG-groupoid with left identity. Then we have

(1) $(a S) a^{2}=(a S) a$.

(2) $(a S)((a S) a)=(a S) a$.

(3) $S((a S) a)=(a S) a$.

(4) $(S a)(a S)=a(a S)$.

(5) $(a S)(S a)=(a S) a$.

(6) $(a(a S)) S=(a S) a$.

(7) $((S a) S)(S a)=(a S)(S a)$.

(8) $(S a) S=(a S)$.

(9) $S(S a)=S a$.

(10) $S a^{2}=a^{2} S$.

Proof. The proof is straightforward and thus omitted.

\section{$3 \quad$ Fuzzy sets and fuzzy soft sets}

The theory of fuzzy sets [1] provides an efficient framework for mathematical description of uncertain concepts. Recall that a fuzzy subset $f$ of a given set $S$ is defined by (and usually identified with) its membership function $f$ : $S \rightarrow[0,1]$. For $x \in S$, the membership value $f(x)$ essentially specifies the degree to which $x \in S$ belongs to the fuzzy subset $f$. By $f \subseteq g$, we mean that $f(x) \leq g(x)$ for all $x \in S$. Clearly $f=g$ if $f \subseteq g$ and $g \subseteq f$. That is, $f(x)=g(x)$ for all $x \in S$.

Let $f$ and $g$ be any fuzzy subsets of an AG-groupoid $S$. Then, the product $f \circ g$ is defined by

$$
(f \circ g)(a)=\left\{\begin{array}{cl}
\bigvee_{a=b c}\{f(b) \wedge g(c)\}, & \text { if there exist } b, c \in S \text { with } a=b c, \\
0, & \text { otherwise. }
\end{array}\right.
$$

A fuzzy subset $f$ of $S$ defined as

$$
f(y)= \begin{cases}t, & \text { if } y=x \\ 0, & \text { otherwise }\end{cases}
$$

is said to be a fuzzy point with support $x$ and value $t$ and is denoted by $x_{t}$, where $t \in(0,1]$.

Let $\gamma, \delta \in[0,1]$ with $\gamma<\delta, X$ be a nonempty set and $Y \subseteq X$. In the sequel, $\chi_{Y}^{(\gamma, \delta)}$ will be used to represent any fuzzy subset of $X$ such that

$$
\chi_{Y}^{(\gamma, \delta)}(x)= \begin{cases}s, & \text { if } x \in Y \\ t, & \text { otherwise }\end{cases}
$$


where $s, t$ are chosen (but fixed) from $[0,1]$ with $s \geq \delta$ and $t \leq \gamma$. It is obvious that $\chi_{Y}^{(0,1)}$ coincides with the characteristic function $\chi_{Y}$ of $Y$. In what follows, $\mathscr{F}(X)$ denotes the set of all fuzzy subsets of $X$.

Let $\gamma, \delta \in[0,1]$ with $\gamma<\delta, x_{r}$ be a fuzzy point and $f \in \mathscr{F}(X)$. Then

(1) $x_{r} \in_{\gamma} f$ if $f(x) \geq r>\gamma$.

(2) $x_{r} q_{\delta} f$ if $f(x)+r>2 \delta$.

(3) $x_{r} \in_{\gamma} \vee q_{\delta} f$ if $x_{r} \in_{\gamma} f$ or $x_{r} q_{\delta} f$.

For $f, g \in \mathscr{F}(X)$, we write $f \subseteq \vee q_{(\gamma, \delta)} g$ if $x_{r} \in_{\gamma} f$ implies $x_{r} \in_{\gamma} \vee q_{\delta} g$ for all $x \in X$ and $r \in(\gamma, 1]$. In addition, $f$ and $g$ are said to be $(\gamma, \delta)$-equal, denoted by $f={ }_{(\gamma, \delta)} g$, if $f \subseteq \vee q_{(\gamma, \delta)} g$ and $g \subseteq \vee q_{(\gamma, \delta)} f$. It is shown in [23] that the " $(\gamma, \delta)$-equal relation" is an equivalence relation on $\mathscr{F}(X)$.

Lemma 4. (see [23]) Let $f$ and $g$ be fuzzy subsets of $X$. Then $f \subseteq \vee q_{(\gamma, \delta)} g$ if and only if $\max \{g(x), \gamma\} \geq \min \{f(x), \delta\}$ for all $x \in X$.

Lemma 5. (see [23]) Let $f, g, h \in \mathscr{F}(X)$. If $f \subseteq \vee q_{(\gamma, \delta)} g$ and $g \subseteq \vee q_{(\gamma, \delta)} h$, then we have $f \subseteq \vee q_{(\gamma, \delta)} h$.

Note that $f={ }_{(\gamma, \delta)} g$ if and only if

$$
\max \{\min \{f(x), \delta\}, \gamma\}=\max \{\min \{g(x), \delta\}, \gamma\}
$$

for all $x \in X$.

Lemma 6. Let $\gamma, \delta \in[0,1]$ with $\gamma<\delta, A$ and $B$ be nonempty subsets of an $A G$-groupoid $S$ with left identity. Then we have

(1) $A \subseteq B$ if and only if $\chi_{A}^{(\gamma, \delta)} \subseteq \vee q_{(\gamma, \delta)} \chi_{B}^{(\gamma, \delta)}$.

(2) $\chi_{A}^{(\gamma, \delta)} \cap \chi_{B}^{(\gamma, \delta)}={ }_{(\gamma, \delta)} \chi_{(A \cap B)}^{(\gamma, \delta)}$.

(3) $\chi_{A}^{(\gamma, \delta)} \circ \chi_{B}^{(\gamma, \delta)}={ }_{(\gamma, \delta)} \chi_{(A B)}^{(\gamma, \delta)}$.

Proof. The proof is straightforward and thus omitted.

Soft set theory was proposed by Molodtsov [4] in 1999, which provides a general mechanism for uncertainty modelling in a wide variety of applications. Let $U$ be an initial universe of objects and $E_{U}$ (or simply $E$ ) be the set of all parameters associated with objects in $U$, called a parameter space. In most cases parameters are considered to be attributes, characteristics or properties of objects in $U$. A pair $\mathfrak{S}=(F, A)$ is called a soft set over $U$, where $A \subseteq E$ and $F: A \rightarrow \mathscr{P}(U)$ is a set-valued mapping, called the approximate function of the soft set $\mathfrak{S}$. By means of parametrization, a soft set produces a series of approximate descriptions of a complicated object being perceived from various points of view. It is apparent that a soft set $\mathfrak{S}=(F, A)$ over a universe $U$ can be viewed as a parameterized family of subsets of $U$.

By combining fuzzy sets with soft set, Maji et al. [24] initiated the following hybrid model called fuzzy soft set. 
Definition 1. (see [24]) A pair $\mathfrak{S}=\langle F, A\rangle$ is called a fuzzy soft set over $U$, where $A \subseteq E$ and $F$ is a mapping given by $F: A \rightarrow \mathscr{F}(U)$.

The mapping $F: A \rightarrow \mathscr{F}(U)$ is called the approximate function of the fuzzy soft set $\langle F, A\rangle$. Given two fuzzy soft sets $\langle F, A\rangle$ and $\langle G, B\rangle$ over $U$, we say that $\langle F, A\rangle$ is a fuzzy soft subset of $\langle G, B\rangle$, denoted $\langle F, A\rangle \subset\langle G, B\rangle$, if $A \subseteq B$ and $F(a)$ is a fuzzy subset of $G(a)$ for all $a \in A$. By $\langle F, A\rangle \supset\langle G, B\rangle$, we mean that $\langle G, B\rangle \subset\langle F, A\rangle$. Moreover, $\langle F, A\rangle$ and $\langle G, B\rangle$ are said to be equal, denoted $\langle F, A\rangle=\langle G, B\rangle$, if $\langle F, A\rangle \subset\langle G, B\rangle$ and $\langle G, B\rangle \subset\langle F, A\rangle$.

A fuzzy soft set $\langle F, A\rangle$ is an $(\gamma, \delta)$-fuzzy soft subset of $\langle G, B\rangle$, denoted by $\langle F, A\rangle \subset_{(\gamma, \delta)}\langle G, B\rangle$, if $A \subseteq B$ and for any $a \in A, F(a) \subseteq \vee q_{(\gamma, \delta)} G(a)$.

The following notions were initially introduced by Ali et al. [25] for Molodtsov's soft sets.

Definition 2. (see [23]) Let $\langle F, A\rangle$ and $\langle G, B\rangle$ be two fuzzy soft sets over $U$.

(1) The extended intersection of $\langle F, A\rangle$ and $\langle G, B\rangle$ is defined as the the fuzzy soft set $\langle H, C\rangle=\langle F, A\rangle \cap_{\mathcal{E}}\langle G, B\rangle$ where $C=A \cup B$ and for all $c \in C$,

$$
H(c)= \begin{cases}F(c), & \text { if } c \in A \backslash B, \\ G(c), & \text { if } c \in B \backslash A, \\ F(c) \cap G(c), & \text { if } c \in A \cap B .\end{cases}
$$

(2) The restricted intersection of $\langle F, A\rangle$ and $\langle G, B\rangle$ is defined as the fuzzy soft set $\langle H, C\rangle=\langle F, A\rangle \cap_{\mathcal{R}}\langle G, B\rangle$, where $C=A \cap B \neq \emptyset$ and $H(c)=$ $F(c) \cap\langle G, B\rangle$ for all $c \in C$.

Let $V$ be a subset of $U$ and $A \subseteq E$. A fuzzy soft set $\langle F, A\rangle$ over $V$ is called a relative whole $(\gamma, \delta)$-fuzzy soft set (with respect to $V$ and $A$ ), denoted by $\Sigma(V, A)$, if $F(a)=\chi_{V}^{(\gamma, \delta)}$ for all $a \in A$.

\section{Fuzzy soft ideals over AG-groupoids}

From now on, unless stated otherwise, let $S$ denote an AG-groupoid.

Definition 3. Let $\langle F, A\rangle$ and $\langle G, B\rangle$ be fuzzy soft sets over $S$.

(1) The extended product of $\langle F, A\rangle$ and $\langle G, B\rangle$ is defined as the the fuzzy soft set $\langle H, C\rangle=\langle F, A\rangle \odot\langle G, B\rangle$, where $C=A \cup B$ and for all $c \in C$,

$$
H(c)= \begin{cases}F(c), & \text { if } c \in A \backslash B, \\ G(c), & \text { if } c \in B \backslash A, \\ F(c) \circ G(c), & \text { if } c \in A \cap B .\end{cases}
$$


(2) The restricted product of $\langle F, A\rangle$ and $\langle G, B\rangle$ is defined as the fuzzy soft set $\langle H, C\rangle=\langle F, A\rangle \boxminus\langle G, B\rangle$, where $C=A \cap B \neq \emptyset$ and $H(c)=F(c) \circ\langle G, B\rangle$ for all $c \in C$.

Proposition 1. Let $\langle F, A\rangle$ and $\langle G, A\rangle$ be fuzzy soft sets over $S$. Then

$$
\langle F, A\rangle \odot\langle G, A\rangle=\langle F, A\rangle \odot\langle G, A\rangle .
$$

Proof. The proof is straightforward and thus omitted.

The above assertion says that the extended product operation $\odot$ coincides with the restricted product $\square$ operation, if the concerned fuzzy soft sets have the same parameter set. Thus for this particular case, we shall use only notation $\odot$ to represent for both operations in what follows.

Definition 4. A fuzzy soft set $\langle F, A\rangle$ over an AG-groupoid $S$ is called

- a fuzzy soft left (resp. right) ideal over $S$ if $\Sigma\langle S, A\rangle \odot\langle F, A\rangle \subset\langle F, A\rangle$ (resp. $\langle F, A\rangle \odot \Sigma\langle S, A\rangle \subset\langle F, A\rangle$ );

- a fuzzy soft bi-ideal over $S$ if $\langle F, A\rangle \odot\langle F, A\rangle \subset\langle F, A\rangle$ and $[\langle F, A\rangle \odot$ $\Sigma\langle S, A\rangle] \odot\langle F, A\rangle \subset\langle F, A\rangle$

- a fuzzy soft generalized bi-ideal over $S$ if $[\langle F, A\rangle \odot \Sigma\langle S, A\rangle] \odot\langle F, A\rangle \subset$ $\langle F, A\rangle$;

- a fuzzy soft quasi-ideal over $S$ if

$$
\left[\langle F, A \odot \Sigma(S, A)] \cap_{\mathcal{E}}[\Sigma(S, A) \odot\langle F, A\rangle] \subset\langle F, A\rangle .\right.
$$

Definition 5. A fuzzy soft set $\langle F, A\rangle$ over an AG-groupoid $S$ is called

- an $\left(\in_{\gamma}, \in_{\gamma} \vee q_{\delta}\right)$-fuzzy soft left ideal over $S$ if $\Sigma(S, A) \odot\langle F, A\rangle \subset_{(\gamma, \delta)}$ $\langle F, A\rangle$;

- an $\left(\in_{\gamma}, \in_{\gamma} \vee q_{\delta}\right)$-fuzzy soft generalized bi-ideal over $S$ if $[\langle F, A\rangle \odot \Sigma(S, A)] \odot$ $\langle F, A\rangle \subset_{(\gamma, \delta)}\langle F, A\rangle$;

- an $\left(\epsilon_{\gamma}, \in_{\gamma} \vee q_{\delta}\right)$-fuzzy soft bi-ideal over $S$ if $\langle F, A\rangle \odot\langle F, A\rangle \subset_{(\gamma, \delta)}\langle F, A\rangle$, and $[\langle F, A\rangle \odot \Sigma(S, A)] \odot\langle F, A\rangle \subset_{(\gamma, \delta)}\langle F, A\rangle$;

- an $\left(\epsilon_{\gamma}, \in_{\gamma} \vee q_{\delta}\right)$-fuzzy soft quasi-ideal over $S$ if

$$
\left[\langle F, A \odot \Sigma(S, A)] \cap_{\mathcal{E}}[\Sigma(S, A) \odot\langle F, A\rangle] \subset_{(\gamma, \delta)}\langle F, A\rangle .\right.
$$


Example 1. Let $S=\{1,2,3\}$ and define a binary operation "” on $S$ as follows:

\begin{tabular}{l|lll}
$\cdot$ & 1 & 2 & 3 \\
\hline 1 & 2 & 2 & 3 \\
2 & 3 & 3 & 3 \\
3 & 3 & 3 & 3
\end{tabular}

Then it can be shown that $(S, \cdot)$ is an AG-groupoid.

Let $A=\{0.35,0.4\}$ and $\langle F, A\rangle$ be a fuzzy soft set over $S$ such that

$$
F(a)(x)= \begin{cases}2 a, & \text { if } x \in\{1,2\} \\ 0.4, & \text { otherwise }\end{cases}
$$

where $a \in A$ and $x \in S$. Then by definition, $\langle F, A\rangle$ is an $\left(\in_{0.3}, \in_{0.3} \vee q_{0.4}\right)$ fuzzy soft left ideal over $S$.

Next, let $B=\{0.7,0.8\}$ and define a fuzzy soft set $\langle G, B\rangle$ over $S$ as follows:

$$
G(b)(x)=\left\{\begin{array}{cl}
b, & \text { if } x \in\{1,2\}, \\
0.4, & \text { otherwise }
\end{array}\right.
$$

where $b \in B$ and $x \in S$. Then it is easy to verify that $\langle G, B\rangle$ is an $\left(\in_{0.2}, \in_{0.2}\right.$ $\vee q_{0.4}$ )-fuzzy soft bi-ideal over $S$.

Theorem 1. For an AG-groupoid $S$ with left identity, the following are equivalent:

(i) $S$ is regular.

(ii) $B[a] \cap I[a] \cap L[a] \subseteq(B[a] I[a]) L[a]$, for some $a$ in $S$.

(iii) For any bi-ideal $B$, ideal $I$ and left ideal $L$ of $S, B \cap I \cap L \subseteq(B I) L$.

(iv) For any $\left(\in_{\gamma}, \in_{\gamma} \vee q_{\delta}\right)$-fuzzy soft bi-ideal $\langle F, A\rangle,\left(\in_{\gamma}, \in_{\gamma} \vee q_{\delta}\right)$-fuzzy soft ideal $\langle G, A\rangle$ and $\left(\in_{\gamma}, \in_{\gamma} \vee q_{\delta}\right)$-fuzzy soft left ideal $\langle H, B\rangle$ over $S$, we have

$$
\langle F, A\rangle \cap_{\mathcal{E}}\langle G, A\rangle \cap_{\mathcal{E}}\langle H, B\rangle \subset_{(\gamma, \delta)}(\langle F, A\rangle \odot\langle G, A\rangle) \odot\langle H, B\rangle .
$$

(v) For any $\left(\in_{\gamma}, \in_{\gamma} \vee q_{\delta}\right)$-fuzzy soft generalized bi-ideal $\langle F, A\rangle,\left(\in_{\gamma}, \in_{\gamma}\right.$ $\left.\vee q_{\delta}\right)$-fuzzy soft ideal $\langle G, A\rangle$ and $\left(\in_{\gamma}, \in_{\gamma} \vee q_{\delta}\right)$-fuzzy soft left ideal $\langle H, B\rangle$ over $S$, we have

$$
\langle F, A\rangle \cap_{\mathcal{E}}\langle G, A\rangle \cap_{\mathcal{E}}\langle H, B\rangle \subset_{(\gamma, \delta)}(\langle F, A\rangle \odot\langle G, A\rangle) \odot\langle H, B\rangle .
$$

(vi) For any $\left(\in_{\gamma}, \in_{\gamma} \vee q_{\delta}\right)$-fuzzy soft generalized bi-ideal $\langle F, A\rangle,\left(\in_{\gamma}, \in_{\gamma}\right.$ $\left.\vee q_{\delta}\right)$-fuzzy soft right ideal $\langle G, A\rangle$ and $\left(\in_{\gamma}, \in_{\gamma} \vee q_{\delta}\right)$-fuzzy soft left ideal $\langle H, B\rangle$ of $S$, we have

$$
\langle F, A\rangle \cap_{\mathcal{E}}\langle G, A\rangle \cap_{\mathcal{E}}\langle H, B\rangle \subset_{(\gamma, \delta)}(\langle F, A\rangle \odot\langle G, A\rangle) \odot\langle H, B\rangle .
$$


Proof. (i) $\Rightarrow(v i)$ Assume that (i) holds. Let $\langle F, A\rangle,\langle G, A\rangle$ and $\langle H, B\rangle$ be any $\left(\epsilon_{\gamma}, \in_{\gamma} \vee q_{\delta}\right)$-fuzzy soft bi-ideal, $\left(\in_{\gamma}, \in_{\gamma} \vee q_{\delta}\right)$-fuzzy soft right ideal and $\left(\in_{\gamma}, \in_{\gamma} \vee q_{\delta}\right)$-fuzzy soft left ideal over $S$, respectively. Let $a$ be any element of $S,\langle F, A\rangle \cap_{\mathcal{E}}\langle G, A\rangle \cap_{\mathcal{E}}\langle H, B\rangle=\left\langle K_{1}, A \cup B\right\rangle$ and $(\langle F, A\rangle \odot\langle G, A\rangle) \odot\langle H, B\rangle=$ $\left\langle K_{2}, A \cup B\right\rangle$.For any $\epsilon \in A \cup B$. We consider the following cases,

Case 1: $\epsilon \in A-B$. Then $K_{1}(\epsilon)=F(\epsilon) \cap G(\epsilon)$ and $K_{2}(\epsilon)=(F \circ G)(\epsilon)$ So, we have $K_{1}(\epsilon) \subseteq \vee q_{(\gamma, \delta)} K_{2}(\epsilon)$

Case 2: $\epsilon \in B-A$. Then $K_{1}(\epsilon)=H(\epsilon)$ and $K_{1}(\epsilon)=H(\epsilon)=K_{2}(\epsilon)$

Case 3: $\epsilon \in A \cap B$. Then $K_{1}(\epsilon)=F(\epsilon) \cap G(\epsilon) \cap H(\epsilon)$ and $K_{2}(\epsilon)=(F(\epsilon) \circ$ $G(\epsilon)) \circ H(\epsilon)$. Now we show that $F(\epsilon) \cap G(\epsilon) \cap H(\epsilon) \subseteq \vee q_{(\gamma, \delta)}(F(\epsilon) \circ G(\epsilon)) \circ H(\epsilon)$. Now since $S$ is regular AG-groupoid, so for $a \in S$ there exist $x \in S$ such that using left invertive law and also using law $a(b c)=b(a c)$, we have,

$$
\begin{aligned}
a & =(a x) a=[\{(a x) a\} x] a=(a x)\{(a x) a\}=[\{(a x) a\} x]\{(a x) a\} \\
& =\{(x a)(a x)\}\{(a x) a\}=[\{(a x) a\}(a x)](x a)
\end{aligned}
$$

Thus we have,

$$
\begin{aligned}
& \max \{((F(\epsilon) \circ G(\epsilon)) \circ H(\epsilon))(a), \gamma\} \\
= & \max \left\{\bigvee_{a=p q}(F(\epsilon) \circ G(\epsilon))(p) \wedge H(\epsilon)(q), \gamma\right\} \\
\geq & \max \{(F(\epsilon) \circ G(\epsilon))[\{(a x) a\}(a x)] \wedge H(\epsilon))(x a), \gamma\} \\
= & \left.\max \left\{\bigvee_{[\{(a x) a\}(a x)]=u v}(F(\epsilon)(u) \wedge G(\epsilon)(v)) \wedge H(\epsilon)(x a)\right\}, \gamma\right\} \\
\geq & \max \{F(\epsilon)\{(a x) a\} \wedge G(\epsilon)(a x) \wedge H(\epsilon)(x a), \gamma\} \\
= & \min \{\max \{F(\epsilon)\{(a x) a\}, \gamma\}, \max \{G(\epsilon)(a x), \gamma\}, \max \{H(\epsilon)(x a), \gamma\}\} \\
\geq & \min \{\min \{(F(\epsilon)(a), \delta\}, \min \{G(\epsilon))(a), \delta\}, \min \{H(\epsilon)(a), \delta\}\} \\
= & \min \{(F(\epsilon) \wedge G(\epsilon) \wedge H(\epsilon))(a), \delta\} \\
= & \min \{(F(\epsilon) \cap G(\epsilon) \cap H(\epsilon))(a), \delta\}
\end{aligned}
$$

Thus $\min \{(F(\epsilon) \cap G(\epsilon) \cap H(\epsilon))(a), \delta\} \leq \max \{((F(\epsilon) \circ G(\epsilon)) \circ H(\epsilon))(a), \gamma\}$. This implies that $F(\epsilon) \cap G(\epsilon) \cap H(\epsilon) \subseteq \vee q_{(\gamma, \delta)}(F(\epsilon) \circ G(\epsilon)) \circ H(\epsilon)$.

Therefore in any case, we have $K_{1}(\epsilon) \subseteq \vee q_{(\gamma, \delta)} K_{2}(\epsilon)$. Hence

$$
\langle F, A\rangle \cap_{\mathcal{E}}\langle G, A\rangle \cap_{\mathcal{E}}\langle H, B\rangle \subset_{(\gamma, \delta)}(\langle F, A\rangle \odot\langle G, A\rangle) \odot\langle H, B\rangle .
$$

$(v i) \Rightarrow(v)$ is obvious.

$(v) \Rightarrow(i v)$ is obvious.

$(i v) \Rightarrow(i i i)$ 
Assume that $B, I$ and $L$ are bi-ideal, ideal and left ideal of $S$, respectively, then $\Sigma(B, E), \Sigma(I, E)$ and $\Sigma(L, E)$ are $\left(\in_{\gamma}, \in_{\gamma} \vee q_{\delta}\right)$-fuzzy soft bi-ideal, $\left(\in_{\gamma}, \in_{\gamma}\right.$ $\left.\vee q_{\delta}\right)$-fuzzy soft ideal and $\left(\epsilon_{\gamma}, \in_{\gamma} \vee q_{\delta}\right)$-fuzzy soft left ideal over $S$, respectively. Now we have assume that $(i v)$ holds, then we have

$$
\Sigma(B, E) \cap_{\mathcal{E}} \Sigma(I, E) \cap_{\mathcal{E}} \Sigma(L, E) \subset_{(\gamma, \delta)}(\Sigma(B, E) \odot \Sigma(I, E)) \odot \Sigma(L, E) .
$$

So,

$$
\begin{array}{rlrl}
\chi_{(B \cap I \cap L)}^{(\gamma, \delta)} & ={ }_{(\gamma, \delta)} & & \chi_{B}^{(\gamma, \delta)} \cap \chi_{I}^{(\gamma, \delta)} \cap \chi_{I}^{(\gamma, \delta)} \\
& \subseteq \vee q_{(\gamma, \delta)} & \left(\chi_{B}^{(\gamma, \delta)} \circ \chi_{I}^{(\gamma, \delta)}\right) \circ \chi_{\gamma L}^{(\gamma, \delta)} \\
& ={ }_{(\gamma, \delta)} & & \chi_{B I}^{(\gamma, \delta)} \circ \chi_{\gamma L}^{(\gamma, \delta)} \\
& ={ }_{(\gamma, \delta)} & & \chi_{(B I) L}^{(\gamma, \delta)} .
\end{array}
$$

Thus $B \cap I \cap L \subseteq(B I) L$.

(iii) $\Rightarrow($ ii $)$ is obvious.

$($ ii $) \Rightarrow($ i $)$

Suppose that $B[a]=a \cup a^{2} \cup(a S) a, I[a]=a \cup S a \cup a S$ and $L[a]=a \cup S a$ are principle bi-ideal, principle ideal and principle left ideal of $S$ generated by $a$ respectively. Thus by (ii), Lemma 3 , left invertive law, paramedial law and using law $a(b c)=b(a c)$, we have,

$$
\begin{aligned}
& \left(a \cup a^{2} \cup(a S) a\right) \cap(a \cup S a \cup a S) \cap(a \cup S a) \\
\subseteq & \left(\left(a \cup a^{2} \cup(a S) a\right)(a \cup S a \cup a S)\right) \\
& (a \cup S a) \\
\subseteq & \{S(a \cup S a \cup a S)\}(a \cup S a) \\
\subseteq & \{S a \cup S(S a) \cup S(a S)\}(a \cup S a) \\
= & (S a \cup a S)(a \cup S a) \\
= & (S a) a \cup(S a)(S a) \cup(a S) a \cup(a S)(S a) \\
= & a^{2} S \cup a^{2} S \cup(a S) a \cup(a S) a \\
= & a^{2} S \cup(a S) a .
\end{aligned}
$$

Hence $S$ is regular.

Theorem 2. For an AG-groupoid $S$ with left identity, the following are equivalent:

(i) $S$ is regular.

(ii) $L[a] \cap I[a] \cap Q[a] \subseteq(L[a] I[a]) Q[a]$, for some $a$ in $S$.

(iii) For any left ideal $L$, ideal $I$ and quasi-ideal $Q$ of $S, L \cap I \cap Q \subseteq(L I) Q$ 
(iv) For any $\left(\in_{\gamma}, \in_{\gamma} \vee q_{\delta}\right)$-fuzzy soft left ideal $\langle F, A\rangle,\left(\in_{\gamma}, \in_{\gamma} \vee q_{\delta}\right)$-fuzzy soft ideal $\langle G, A\rangle$ and $\left(\in_{\gamma}, \in_{\gamma} \vee q_{\delta}\right)$-fuzzy soft quasi-ideal $\langle H, B\rangle$ over $S$, we have

$$
\langle F, A\rangle \cap_{\mathcal{E}}\langle G, A\rangle \cap_{\mathcal{E}}\langle H, B\rangle \subset_{(\gamma, \delta)}(\langle F, A\rangle \odot\langle G, A\rangle) \odot\langle H, B\rangle .
$$

(v) For any $\left(\in_{\gamma}, \in_{\gamma} \vee q_{\delta}\right)$-fuzzy soft left ideal $\langle F, A\rangle,\left(\in_{\gamma}, \in_{\gamma} \vee q_{\delta}\right)$-fuzzy soft right ideal $\langle G, A\rangle$ and $\left(\in_{\gamma}, \in_{\gamma} \vee q_{\delta}\right)$-fuzzy soft quasi-ideal $\langle H, B\rangle$ over $S$, we have

$$
\langle F, A\rangle \cap_{\mathcal{E}}\langle G, A\rangle \cap_{\mathcal{E}}\langle H, B\rangle \subset_{(\gamma, \delta)}(\langle F, A\rangle \odot\langle G, A\rangle) \odot\langle H, B\rangle .
$$

Proof. $(i) \Rightarrow(v)$ Assume that $\langle F, A\rangle,\langle G, A\rangle$ and $\langle H, B\rangle$ are $\left(\in_{\gamma}, \in_{\gamma} \vee q_{\delta}\right)$-fuzzy soft left ideal, $\left(\epsilon_{\gamma}, \in_{\gamma} \vee q_{\delta}\right)$-fuzzy soft right ideal and $\left(\epsilon_{\gamma}, \in_{\gamma} \vee q_{\delta}\right)$-fuzzy soft quasi-ideal of a regular AG-groupoid $S$, respectively. Let $a$ be any element of $S,\langle F, A\rangle \cap_{\mathcal{E}}\langle G, A\rangle \tilde{\cap}\langle H, B\rangle=\left\langle K_{1}, A \cup B\right\rangle$ and $(\langle F, A\rangle \odot\langle G, A\rangle) \odot\langle H, B\rangle=$ $\left\langle K_{2}, A \cup B\right\rangle$.For any $\epsilon \in A \cup B$. We consider the following cases,

Case 1: $\epsilon \in A-B$. Then $K_{1}(\epsilon)=F(\epsilon) \cap G(\epsilon)$ and $K_{2}(\epsilon)=(F \circ G)(\epsilon)$ So, we have $K_{1}(\epsilon) \subseteq \vee q_{(\gamma, \delta)} K_{2}(\epsilon)$

Case 2: $\epsilon \in B-A$.Then $K_{1}(\epsilon)=H(\epsilon)$ and $K_{1}(\epsilon)=H(\epsilon)=K_{2}(\epsilon)$

Case 3: $\epsilon \in A \cap B$.Then $K_{1}(\epsilon)=F(\epsilon) \cap G(\epsilon) \cap H(\epsilon)$ and $K_{2}(\epsilon)=(F(\epsilon) \circ$ $G(\epsilon)) \circ H(\epsilon)$. Now we show that $F(\epsilon) \cap G(\epsilon) \cap H(\epsilon) \subseteq \vee q_{(\gamma, \delta)}(F(\epsilon) \circ G(\epsilon)) \circ H(\epsilon)$ Now since $S$ is regular, so for $a \in S$ there exist $x \in S$ such that using left invertive law and also using law $a(b c)=b(a c)$, we have,

$$
a=(a x) a=[\{(a x) a\} x] a=\{(x a)(a x)\} a .
$$

Thus

$$
\begin{aligned}
& \max \{((F(\epsilon) \circ G(\epsilon)) \circ H(\epsilon))(a), \gamma\} \\
= & \max \left\{\bigvee_{a=p q}(F(\epsilon) \circ G(\epsilon))(p) \wedge H(\epsilon)(q), \gamma\right\} \\
\geq & \max \{(F(\epsilon) \circ G(\epsilon))[(x a)(a x)] \wedge H(\epsilon))(a), \gamma\} \\
= & \left.\max \left\{\bigvee_{(x a)(a x)=u v}(F(\epsilon)(u) \wedge G(\epsilon)(v)) \wedge H(\epsilon)(a)\right\}, \gamma\right\} \\
\geq & \max \{F(\epsilon)(x a) \wedge G(\epsilon)(a x) \wedge H(\epsilon)(a), \gamma\} \\
= & \min \{\max \{F(\epsilon)(x a), \gamma\}, \max \{G(\epsilon)(a x), \gamma\}, \max \{H(\epsilon)(a), \gamma\}\} \\
\geq & \min \{\min \{(F(\epsilon)(a), \delta\}, \min \{G(\epsilon))(a), \delta\}, \min \{H(\epsilon)(a), \delta\}\} \\
= & \min \{(F(\epsilon) \wedge G(\epsilon) \wedge H(\epsilon))(a), \delta\} \\
= & \min \{(F(\epsilon) \cap G(\epsilon) \cap H(\epsilon))(a), \delta\}
\end{aligned}
$$


Thus we deduce

$\min \{(F(\epsilon) \cap G(\epsilon) \cap H(\epsilon))(a), \delta\} \leq \max \{((F(\epsilon) \circ G(\epsilon)) \circ H(\epsilon))(a), \gamma\}$,

which implies that $F(\epsilon) \cap G(\epsilon) \cap H(\epsilon) \subseteq \vee q_{(\gamma, \delta)}(F(\epsilon) \circ G(\epsilon)) \circ H(\epsilon)$.

Therefore in any case, we have $K_{1}(\epsilon) \subseteq \vee q_{(\gamma, \delta)} K_{2}(\epsilon)$. Hence

$$
\langle F, A\rangle \cap_{\mathcal{E}}\langle G, A\rangle \cap_{\mathcal{E}}\langle H, B\rangle \subset_{(\gamma, \delta)}(\langle F, A\rangle \odot\langle G, A\rangle) \odot\langle H, B\rangle .
$$

$(v) \Rightarrow(i v)$ is obvious.

$(i v) \Rightarrow(i i i)$

Assume that $L, I$ and $Q$ are left ideal, ideal and quasi-ideal of $S$ respectively, then $\Sigma(L, E), \Sigma(I, E)$ and $\Sigma(Q, E)$ are $\left(\epsilon_{\gamma}, \in_{\gamma} \vee q_{\delta}\right)$-fuzzy soft left ideal, $\left(\epsilon_{\gamma}, \in_{\gamma} \vee q_{\delta}\right)$-fuzzy soft ideal and $\left(\epsilon_{\gamma}, \in_{\gamma} \vee q_{\delta}\right)$-fuzzy soft quasi-ideal over $S$, respectively. Now we have assume that $(i v)$ holds, then we have

$$
\Sigma(L, E) \cap_{\mathcal{\varepsilon}} \Sigma(I, E) \cap_{\mathcal{\varepsilon}} \Sigma(Q, E) \subset_{(\gamma, \delta)}(\Sigma(L, E) \odot \Sigma(I, E)) \odot \Sigma(Q, E) .
$$

So, we get

$$
\begin{aligned}
\chi_{(L \cap I \cap Q)}^{(\gamma, \delta)} & ={ }_{(\gamma, \delta)} \quad \chi_{L}^{(\gamma, \delta)} \cap \chi_{I}^{(\gamma, \delta)} \cap \chi_{Q}^{(\gamma, \delta)} \subseteq \vee q_{(\gamma, \delta)}\left(\chi_{L}^{\delta} \circ \chi_{I}^{(\gamma, \delta)}\right) \circ \chi_{Q}^{(\gamma, \delta)} \\
& ={ }_{(\gamma, \delta)} \quad\left(\chi_{L I}^{(\gamma, \delta)}\right) \circ \chi_{Q}^{(\gamma, \delta)}={ }_{(\gamma, \delta)} \chi_{(L I) Q}^{(\gamma, \delta)} .
\end{aligned}
$$

Therefore $L \cap I \cap Q \subseteq(L I) Q$.

$($ iii $) \Rightarrow($ ii $)$ is obvious.

(ii) $\Rightarrow i$

$L[a]=a \cup S a, I[a]=a \cup S a \cup a S$ and $Q[a]=a \cup(S a \cap a S)$ are left ideal, ideal and quasi-ideal of $S$ generated $a$ respectively. Thus by (ii), Lemma 3 and medial law we have,

$$
\begin{aligned}
& (a \cup S a) \cap(a \cup S a \cup a S) \cap(a \cup(S a \cap a S)) \\
\subseteq & ((a \cup S a)(a \cup S a \cup a S)) \\
& (a \cup(S a \cap a S)) \\
\subseteq & \{(a \cup S a) S\}(a \cup a S) \\
= & \{a S \cup(S a) S\}(a \cup a S) \\
= & (a S)(a \cup a S) \\
= & (a S) a \cup(a S)(a S) \\
= & (a S) a \cup a^{2} S .
\end{aligned}
$$

Hence $S$ is regular. 
Theorem 3. For an AG-groupoid $S$ with left identity, the following are equivalent:

(i) $S$ is regular.

(ii) $B[a] \cap I[a] \cap Q[a] \subseteq(B[a] I[a]) Q[a]$, for some a in $S$.

(iii) For any bi-ideal $B$, ideal $I$ and quasi-ideal $Q$ of $S, B \cap I \cap Q \subseteq(B I) Q$.

(iv) For any $\left(\in_{\gamma}, \in_{\gamma} \vee q_{\delta}\right)$-fuzzy soft bi-ideal $\langle F, A\rangle,\left(\in_{\gamma}, \in_{\gamma} \vee q_{\delta}\right)$-fuzzy soft ideal $\langle G, A\rangle$ and $\left(\in_{\gamma}, \in_{\gamma} \vee q_{\delta}\right)$-fuzzy soft quasi-ideal $\langle H, B\rangle$ over $S$, we have

$$
\langle F, A\rangle \cap_{\mathcal{E}}\langle G, A\rangle \cap_{\mathcal{E}}\langle H, B\rangle \subset_{(\gamma, \delta)}(\langle F, A\rangle \odot\langle G, A\rangle) \odot\langle H, B\rangle .
$$

(v) For any $\left(\in_{\gamma}, \in_{\gamma} \vee q_{\delta}\right)$-fuzzy soft generalized bi-ideal $\langle F, A\rangle,\left(\in_{\gamma}, \in_{\gamma}\right.$ $\left.\vee q_{\delta}\right)$-fuzzy soft ideal $\langle G, A\rangle$ and $\left(\in_{\gamma}, \in_{\gamma} \vee q_{\delta}\right)$-fuzzy soft quasi-ideal $\langle H, B\rangle$ over $S$, we have

$$
\langle F, A\rangle \cap_{\mathcal{E}}\langle G, A\rangle \cap_{\mathcal{E}}\langle H, B\rangle \subset_{(\gamma, \delta)}(\langle F, A\rangle \odot\langle G, A\rangle) \odot\langle H, B\rangle .
$$

Proof. $(i) \Rightarrow(v)$ Assume that $\langle F, A\rangle,\langle G, A\rangle$ and $\langle H, B\rangle$ are $\left(\epsilon_{\gamma}, \epsilon_{\gamma} \vee q_{\delta}\right)$-fuzzy soft bi- ideal, $\left(\epsilon_{\gamma}, \in_{\gamma} \vee q_{\delta}\right)$-fuzzy soft ideal and $\left(\epsilon_{\gamma}, \in_{\gamma} \vee q_{\delta}\right)$-fuzzy soft quasiideal of a regular AG-groupoid $S$, respectively. Let $a$ be any element of $S$, $\langle F, A\rangle \cap_{\mathcal{E}}\langle G, A\rangle \tilde{\cap}\langle H, B\rangle=\left\langle K_{1}, A \cup B\right\rangle$ and $(\langle F, A\rangle \odot\langle G, A\rangle) \odot\langle H, B\rangle=$ $\left\langle K_{2}, A \cup B\right\rangle$. For any $\epsilon \in A \cup B$. We consider the following cases,

Case 1: $\epsilon \in A-B$. Then $K_{1}(\epsilon)=F(\epsilon) \cap G(\epsilon)$ and $K_{2}(\epsilon)=(F \circ G)(\epsilon)$ So, we have $K_{1}(\epsilon) \subseteq \vee q_{(\gamma, \delta)} K_{2}(\epsilon)$

Case 2: $\epsilon \in B-A$.Then $K_{1}(\epsilon)=H(\epsilon)$ and $K_{1}(\epsilon)=H(\epsilon)=K_{2}(\epsilon)$

Case 3: $\epsilon \in A \cap B$. Then $K_{1}(\epsilon)=F(\epsilon) \cap G(\epsilon) \cap H(\epsilon)$ and $K_{2}(\epsilon)=(F(\epsilon) \circ$ $G(\epsilon)) \circ H(\epsilon)$. First we show that $F(\epsilon) \cap G(\epsilon) \cap H(\epsilon) \subseteq \vee q_{(\gamma, \delta)}(F(\epsilon) \circ G(\epsilon)) \circ H(\epsilon)$. Now since $S$ is regular, so for $a \in S$ there exist $x \in S$ such that by using left invertive law and also using law $a(b c)=b(a c)$, we have,

$$
a=(a x) a=(((a x) a) x) a=((x a)(a x)) a=[a\{(x a) x\}] a .
$$


Thus

$$
\begin{aligned}
& \max \{((F(\epsilon) \circ G(\epsilon)) \circ H(\epsilon))(a), \gamma\} \\
= & \max \left\{\bigvee_{a=x y}(F(\epsilon) \circ G(\epsilon))(x) \wedge H(\epsilon)(y), \gamma\right\} \\
\geq & \max \{(F(\epsilon) \circ G(\epsilon))[a\{(x a) x\}] \wedge H(\epsilon))(a), \gamma\} \\
= & \left.\max \left\{\bigvee_{[a\{(x a) x\}]=p q}(F(\epsilon)(p) \wedge G(\epsilon)(q)) \wedge H(\epsilon)(a)\right\}, \gamma\right\} \\
\geq & \max \{F(\epsilon)(a) \wedge G(\epsilon)\{(x a) x\} \wedge H(\epsilon)(a), \gamma\} \\
= & \min \{\max \{F(\epsilon)(a), \gamma\}, \max \{G(\epsilon)\{(x a) x\}, \gamma\}, \max \{H(\epsilon)(a), \gamma\}\} \\
\geq & \min \{\min \{(F(\epsilon)(a), \delta\}, \min \{G(\epsilon))(a), \delta\}, \min \{H(\epsilon)(a), \delta\}\} \\
= & \min \{(F(\epsilon) \wedge G(\epsilon) \wedge H(\epsilon))(a), \delta\} \\
= & \min \{(F(\epsilon) \cap G(\epsilon) \cap H(\epsilon))(a), \delta\}
\end{aligned}
$$

Thus we have

$$
\min \{(F(\epsilon) \cap G(\epsilon) \cap H(\epsilon))(a), \delta\} \leq \max \{((F(\epsilon) \circ G(\epsilon)) \circ H(\epsilon))(a), \gamma\},
$$

which implies that $F(\epsilon) \cap G(\epsilon) \cap H(\epsilon) \subseteq \vee q_{(\gamma, \delta)}(F(\epsilon) \circ G(\epsilon)) \circ H(\epsilon)$.

Therefore in any case, we have $K_{1}(\epsilon) \subseteq \vee q_{(\gamma, \delta)} K_{2}(\epsilon)$. Hence

$$
\langle F, A\rangle \cap_{\mathcal{E}}\langle G, A\rangle \cap_{\mathcal{E}}\langle H, B\rangle \subset_{(\gamma, \delta)}(\langle F, A\rangle \odot\langle G, A\rangle) \odot\langle H, B\rangle .
$$

$(v) \Rightarrow(i v)$ is obvious.

(iv) $\Rightarrow($ iii $)$

Assume that $B, I$ and $Q$ are bi-ideal, ideal and quasi-ideal of regular AGgroupioud of $S$ respectively, then $\Sigma(B, E), \Sigma(I, E)$ and $\Sigma(Q, E)$ are $\left(\epsilon_{\gamma}, \in_{\gamma}\right.$ $\left.\vee q_{\delta}\right)$-fuzzy soft bi-ideal, $\left(\epsilon_{\gamma}, \in_{\gamma} \vee q_{\delta}\right)$-fuzzy soft ideal and $\left(\epsilon_{\gamma}, \in_{\gamma} \vee q_{\delta}\right)$-fuzzy soft quasi-ideal over $S$,respectively. Now we have assume that $(i v)$ holds, then we have

$\Sigma(B, E) \cap_{\mathcal{E}} \Sigma(I, E) \cap_{\mathcal{E}} \Sigma(Q, E) \subset_{(\gamma, \delta)}(\Sigma(B, E) \odot \Sigma(I, E)) \odot \Sigma(Q, E)$.

Thus it follows that

$$
\begin{aligned}
\chi_{(B \cap I \cap Q)}^{(\gamma, \delta)} & ={ }_{(\gamma, \delta)} \chi_{L}^{(\gamma, \delta)} \cap \chi_{I}^{(\gamma, \delta)} \cap \chi_{Q}^{(\gamma, \delta)} \subseteq \vee q_{(\gamma, \delta)}\left(\chi_{B}^{\delta} \circ \chi_{I}^{(\gamma, \delta)}\right) \circ \chi_{Q}^{(\gamma, \delta)} \\
& ={ }_{(\gamma, \delta)}\left(\chi_{B I}^{(\gamma, \delta)}\right) \circ \chi_{Q}^{(\gamma, \delta)}={ }_{(\gamma, \delta)} \chi_{(B I) Q}^{(\gamma, \delta)} .
\end{aligned}
$$

Therefore $B \cap I \cap Q \subseteq(B I) Q$.

$($ iii $) \Rightarrow($ ii $)$ is obvious. 
$(i i) \Rightarrow(i)$

Since $B[a]=a \cup a^{2} \cup(a S) a, I[a]=a \cup S a \cup a S$ and $Q[a]=a \cup(S a \cap a S)$ are principle bi-ideal, principle ideal and principle quasi-ideal of $S$ generated by $a$ respectively. Thus by $(i i)$ and Lemma 3 , using law $a(b c)=b(a c)$, medial law and left invertive law, we have,

$$
\begin{aligned}
& \left(a \cup a^{2} \cup(a S) a\right) \cap(a \cup S a \cup a S) \cap(a \cup(S a \cap a S)) \\
\subseteq & \left(\left(a \cup a^{2} \cup(a S) a\right)(a \cup S a\right. \\
& \cup a S))(a \cup(S a \cap a S)) \\
\subseteq & (S(a \cup S a \cup a S))(a \cup a S) \\
= & (S a \cup S(S a) \cup S(a S))(a \cup a S) \\
= & (S a \cup S(S a) \cup S(a S))(a \cup a S) \\
= & (a S \cup S a)(a \cup a S) \\
= & (a S) a \cup(a S)(a S) \cup(S a) a \cup(S a)(a S) \\
= & (a S) a \cup a^{2} S \cup a(a S) .
\end{aligned}
$$

Hence $S$ is regular.

Theorem 4. For an AG-groupoid $S$ with left identity, the following are equivalent:

(i) $S$ is regular.

(ii) $I[a] \cap I[a] \cap I[a] \subseteq(I[a] I[a]) I[a]$, for some $a$ in $S$.

(iii) For any ideals $I_{1}, I_{2}$ and $I_{3}$ of $S, I_{1} \cap I_{2} \cap I_{3} \subseteq\left(I_{1} I_{2}\right) I_{3}$. then

(iv) If $\langle F, A\rangle,\langle G, A\rangle$ and $\langle H, B\rangle$ are $\left(\in_{\gamma}, \in_{\gamma} \vee q_{\delta}\right)$-fuzzy soft ideals over $S$,

$$
\langle F, A\rangle \cap_{\mathcal{E}}\langle G, A\rangle \cap_{\mathcal{E}}\langle H, B\rangle \subset_{(\gamma, \delta)}(\langle F, A\rangle \odot\langle G, A\rangle) \odot\langle H, B\rangle .
$$

Proof. $(i) \Rightarrow(i v)$

Assume that $\langle F, A\rangle,\langle G, A\rangle$ and $\langle H, B\rangle$ are any $\left(\epsilon_{\gamma}, \in_{\gamma} \vee q_{\delta}\right)$-fuzzy soft ideals over a regular AG-groupoid $S$, respectively. Let $a$ be any element of $S,\langle F, A\rangle \cap_{\mathcal{E}}\langle G, A\rangle \tilde{\cap}\langle H, B\rangle=\left\langle K_{1}, A \cup B\right\rangle$ and $(\langle F, A\rangle \odot\langle G, A\rangle) \odot\langle H, B\rangle=$ $\left\langle K_{2}, A \cup B\right\rangle$. For any $\epsilon \in A \cup B$. We consider the following cases,

Case 1: $\epsilon \in A-B$. Then $K_{1}(\epsilon)=F(\epsilon) \cap G(\epsilon)$ and $K_{2}(\epsilon)=(F \circ G)(\epsilon)$. So we have $K_{1}(\epsilon) \subseteq \vee q_{(\gamma, \delta)} K_{2}(\epsilon)$.

Case 2: $\epsilon \in B-A$. Then $K_{1}(\epsilon)=H(\epsilon)$ and $K_{1}(\epsilon)=H(\epsilon)=K_{2}(\epsilon)$.

Case 3: $\epsilon \in A \cap B$. Then $K_{1}(\epsilon)=F(\epsilon) \cap G(\epsilon) \cap H(\epsilon)$ and $K_{2}(\epsilon)=(F(\epsilon) \circ$ $G(\epsilon)) \circ H(\epsilon)$. First we show that $F(\epsilon) \cap G(\epsilon) \cap H(\epsilon) \subseteq \vee q_{(\gamma, \delta)}(F(\epsilon) \circ G(\epsilon)) \circ H(\epsilon)$. Now since $S$ is regular, so for $a \in S$ there exist $x \in S$ such that by using left invertive law and also using law $a(b c)=b(a c)$, we have,

$$
a=(a x) a=[\{(a x) a\} x] a=((x a)(a x)) a .
$$


Thus

$$
\begin{aligned}
& \max \{((F(\epsilon) \circ G(\epsilon)) \circ H(\epsilon))(a), \gamma\} \\
= & \max \left\{\bigvee_{a=u v}(F(\epsilon) \circ G(\epsilon))(u) \wedge H(\epsilon)(v), \gamma\right\} \\
\geq & \max \{(F(\epsilon) \circ G(\epsilon))[(x a)(a x)] \wedge H(\epsilon))(a), \gamma\} \\
= & \left.\max \left\{\bigvee_{(x a)(a x)=b c}(F(\epsilon)(b) \wedge G(\epsilon)(c)) \wedge H(\epsilon)(a)\right\}, \gamma\right\} \\
\geq & \max \{F(\epsilon)(x a) \wedge G(\epsilon)(a x) \wedge H(\epsilon)(a), \gamma\} \\
= & \min \{\max \{F(\epsilon)(x a), \gamma\}, \max \{G(\epsilon)(a x), \gamma\}, \max \{H(\epsilon)(a), \gamma\}\} \\
\geq & \min \{\min \{(F(\epsilon)(a), \delta\}, \min \{G(\epsilon))(a), \delta\}, \min \{H(\epsilon)(a), \delta\}\} \\
= & \min \{(F(\epsilon) \wedge G(\epsilon) \wedge H(\epsilon))(a), \delta\} \\
= & \min \{(F(\epsilon) \cap G(\epsilon) \cap H(\epsilon))(a), \delta\}
\end{aligned}
$$

Thus min $\{(F(\epsilon) \cap G(\epsilon) \cap H(\epsilon))(a), \delta\} \leq \max \{((F(\epsilon) \circ G(\epsilon)) \circ H(\epsilon))(a), \gamma\}$. This implies that $F(\epsilon) \cap G(\epsilon) \cap H(\epsilon) \subseteq \vee q_{(\gamma, \delta)}(F(\epsilon) \circ G(\epsilon)) \circ H(\epsilon)$.

Therefore in any case, we have $K_{1}(\epsilon) \subseteq \vee q_{(\gamma, \delta)} K_{2}(\epsilon)$. Hence

$$
\langle F, A\rangle \cap_{\mathcal{E}}\langle G, A\rangle \cap_{\mathcal{E}}\langle H, B\rangle \subset_{(\gamma, \delta)}(\langle F, A\rangle \odot\langle G, A\rangle) \odot\langle H, B\rangle .
$$

$(i v) \Rightarrow(i i i)$ is obvious.

Assume that $I_{1}, I_{2}$ and $I_{3}$ are any ideals of regular AG-groupioud of $S$ respectively, then $\Sigma\left(I_{1}, E\right), \Sigma\left(I_{2}, E\right)$ and $\Sigma\left(I_{3}, E\right)$ are $\left(\epsilon_{\gamma}, \epsilon_{\gamma} \vee q_{\delta}\right)$-fuzzy soft ideals over $S$,respectively. Now we have assume that $(i v)$ holds, then we have

$$
\Sigma\left(I_{1}, E\right) \cap_{\mathcal{E}} \Sigma\left(I_{2}, E\right) \cap_{\mathcal{E}} \Sigma\left(I_{3}, E\right) \subset_{(\gamma, \delta)}\left(\Sigma\left(I_{1}, E\right) \odot \Sigma\left(I_{2}, E\right)\right) \odot \Sigma\left(I_{3}, E\right) .
$$

So, we get

$$
\begin{aligned}
\chi_{\left(I_{1} \cap I_{2} \cap I_{3}\right)}^{(\gamma, \delta)} & ={ }_{(\gamma, \delta)} \chi_{I_{1}}^{(\gamma, \delta)} \cap \chi_{I_{2}}^{(\gamma, \delta)} \cap \chi_{I_{3}}^{(\gamma, \delta)} \subseteq \vee q_{(\gamma, \delta)}\left(\chi_{I_{1}}^{(\gamma, \delta)} \circ \chi_{I_{2}}^{(\gamma, \delta)}\right) \circ \chi_{I_{3}}^{(\gamma, \delta)} \\
& ={ }_{(\gamma, \delta)}\left(\chi_{I_{1} I_{2}}^{(\gamma, \delta)}\right) \circ \chi_{I_{3}}^{(\gamma, \delta)}={ }_{(\gamma, \delta)} \chi_{\left(I_{1} I_{2}\right) I_{3}}^{(\gamma, \delta)} .
\end{aligned}
$$

Therefore $I_{1} \cap I_{2} \cap I_{3} \subseteq\left(I_{1} I_{2}\right) I_{3}$.

$($ iii $) \Rightarrow($ ii $)$ is obvious.

$(i i) \Rightarrow(i)$

Since $I[a]=a \cup S a \cup a S$ is a principle ideal of $S$ generated by $a$. Thus by 
(ii), Lemma 3, left invertive law, medial law and paramedial law, we have

$$
\begin{aligned}
& (a \cup S a \cup a S) \cap(a \cup S a \cup a S) \cap(a \cup S a \cup a S) \\
\subseteq & ((a \cup S a \cup a S)(a \cup S a \cup a S))(a \cup S a \cup a S) \\
\subseteq & \{(a \cup S a \cup a S) S\}(a \cup S a \cup a S) \\
= & \{a S \cup(S a) S \cup(a S) S\}(a \cup S a \cup a S) \\
= & \{a S \cup S a\}(a \cup S a \cup a S) \\
= & (a S) a \cup(a S)(S a) \cup(a S)(a S) \cup(S a) a \\
& \cup(S a)(S a) \cup(S a)(a S) \\
= & (a S) a \cup a^{2} S .
\end{aligned}
$$

Hence $S$ is regular.

Theorem 5. For an AG-groupoid $S$ with left identity, the following are equivalent:

(i) $S$ is regular.

(ii) $Q[a] \cap I[a] \cap Q[a] \subseteq(Q[a] I[a]) Q[a]$, for some a in $S$.

(iii) For any quasi-ideals $Q_{1}, Q_{2}$ and ideal $I$ of $S, Q_{1} \cap I \cap Q_{2} \subseteq\left(Q_{1} I\right) Q_{2}$.

(iv) If $\langle F, A\rangle$ and $\langle H, B\rangle$ are $\left(\in_{\gamma}, \in_{\gamma} \vee q_{\delta}\right)$-fuzzy soft quasi-ideals over $S$, and $\langle G, A\rangle$ is a $\left(\in_{\gamma}, \in_{\gamma} \vee q_{\delta}\right)$-fuzzy soft ideal over $S$, then we have

$$
\langle F, A\rangle \cap_{\mathcal{E}}\langle G, A\rangle \cap_{\mathcal{E}}\langle H, B\rangle \subset_{(\gamma, \delta)}(\langle F, A\rangle \odot\langle G, A\rangle) \odot\langle H, B\rangle .
$$

Proof. (i) $\Rightarrow(i v)$ Assume that $\langle F, A\rangle$ and $\langle H, B\rangle$ are any $\left(\epsilon_{\gamma}, \in_{\gamma} \vee q_{\delta}\right)$-fuzzy soft quasi-ideals and $\langle G, A\rangle$ is any $\left(\epsilon_{\gamma}, \in_{\gamma} \vee q_{\delta}\right)$-fuzzy soft ideal of a regular AGgroupoid $S$, respectively. Let $a$ be any element of $S,\langle F, A\rangle \cap_{\mathcal{E}}\langle G, A\rangle \tilde{\cap}\langle H, B\rangle=$ $\left\langle K_{1}, A \cup B\right\rangle$ and $(\langle F, A\rangle \odot\langle G, A\rangle) \odot\langle H, B\rangle=\left\langle K_{2}, A \cup B\right\rangle$. For any $\epsilon \in A \cup B$. We consider the following cases,

Case 1: $\epsilon \in A-B$. Then $K_{1}(\epsilon)=F(\epsilon) \cap G(\epsilon)$ and $K_{2}(\epsilon)=(F \circ G)(\epsilon)$. Hence we have $K_{1}(\epsilon) \subseteq \vee q_{(\gamma, \delta)} K_{2}(\epsilon)$.

Case 2: $\epsilon \in B-A$. Then $K_{1}(\epsilon)=H(\epsilon)$ and $K_{1}(\epsilon)=H(\epsilon)=K_{2}(\epsilon)$.

Case 3: $\epsilon \in A \cap B$. Then $K_{1}(\epsilon)=F(\epsilon) \cap G(\epsilon) \cap H(\epsilon)$ and $K_{2}(\epsilon)=(F(\epsilon) \circ$ $G(\epsilon)) \circ H(\epsilon)$. First we show that $F(\epsilon) \cap G(\epsilon) \cap H(\epsilon) \subseteq \vee q_{(\gamma, \delta)}(F(\epsilon) \circ G(\epsilon)) \circ H(\epsilon)$. Now since $S$ is regular, so for $a \in S$ there exist $x \in S$ such that by using left invertive law and also using law $a(b c)=b(a c)$, we have

$$
a=(a x) a=[\{(a x) a\} x] a=\{(x a)(a x)\} a=[a\{(x a) x\}] a .
$$


Thus

$$
\begin{aligned}
& \max \{((F(\epsilon) \circ G(\epsilon)) \circ H(\epsilon))(a), \gamma\} \\
= & \max \left\{\bigvee_{a=p q}(F(\epsilon) \circ G(\epsilon))(p) \wedge H(\epsilon)(q), \gamma\right\} \\
\geq & \max \{(F(\epsilon) \circ G(\epsilon))[a\{(x a) x\}] \wedge H(\epsilon))(a), \gamma\} \\
= & \left.\max \left\{\bigvee_{[a\{(x a) x\}]=b c}(F(\epsilon)(b) \wedge G(\epsilon)(c)) \wedge H(\epsilon)(a)\right\}, \gamma\right\} \\
\geq & \max \{F(\epsilon)(a) \wedge G(\epsilon)\{(x a) x\} \wedge H(\epsilon)(a), \gamma\} \\
= & \min \{\max \{F(\epsilon)(a), \gamma\}, \max \{G(\epsilon)\{(x a) x\}, \gamma\}, \max \{H(\epsilon)(a), \gamma\}\} \\
\geq & \min \{\min \{(F(\epsilon)(a), \delta\}, \min \{G(\epsilon))(a), \delta\}, \min \{H(\epsilon)(a), \delta\}\} \\
= & \min \{(F(\epsilon) \wedge G(\epsilon) \wedge H(\epsilon))(a), \delta\} \\
= & \min \{(F(\epsilon) \cap G(\epsilon) \cap H(\epsilon))(a), \delta\}
\end{aligned}
$$

Thus $\min \{(F(\epsilon) \cap G(\epsilon) \cap H(\epsilon))(a), \delta\} \leq \max \{((F(\epsilon) \circ G(\epsilon)) \circ H(\epsilon))(a), \gamma\}$. This implies that $F(\epsilon) \cap G(\epsilon) \cap H(\epsilon) \subseteq \vee q_{(\gamma, \delta)}(F(\epsilon) \circ G(\epsilon)) \circ H(\epsilon)$.

Therefore in any case, we have $K_{1}(\epsilon) \subseteq \vee q_{(\gamma, \delta)} K_{2}(\epsilon)$. Hence

$$
\langle F, A\rangle \cap_{\mathcal{E}}\langle G, A\rangle \cap_{\mathcal{E}}\langle H, B\rangle \subset_{(\gamma, \delta)}(\langle F, A\rangle \odot\langle G, A\rangle) \odot\langle H, B\rangle .
$$

$(i v) \Rightarrow\left(\right.$ iii) Assume that $Q_{1}$ and $Q_{2}$ are quasi-ideals and $I$ is an ideal of a regular AG-groupoid $S$., then $\Sigma\left(Q_{1}, E\right)$ and $\Sigma\left(Q_{2}, E\right)$ are $\left(\epsilon_{\gamma}, \in_{\gamma} \vee q_{\delta}\right)$-fuzzy soft quasi-ideals and, $\Sigma(I, E)$ is a $\left(\in_{\gamma}, \in_{\gamma} \vee q_{\delta}\right)$-fuzzy soft ideal over $S$, Now we have assume that $(i v)$ holds, then we have

$$
\Sigma\left(Q_{1}, E\right) \cap_{\mathcal{E}} \Sigma(I, E) \cap_{\mathcal{E}} \Sigma\left(Q_{3}, E\right) \subset_{(\gamma, \delta)}\left(\Sigma\left(Q_{1}, E\right) \odot \Sigma(I, E)\right) \odot \Sigma\left(Q_{2}, E\right) .
$$

So, we get

$$
\begin{aligned}
\chi_{\left(Q_{1} \cap I \cap Q_{2}\right)}^{(\gamma, \delta)} & ={ }_{(\gamma, \delta)} \quad \chi_{Q_{1}}^{(\gamma, \delta)} \cap \chi_{I}^{(\gamma, \delta)} \cap \chi_{Q_{2}}^{(\gamma, \delta)} \subseteq \vee q_{(\gamma, \delta)}\left(\chi_{Q_{1}}^{(\gamma, \delta)} \circ \chi_{I}^{(\gamma, \delta)}\right) \circ \chi_{Q_{2}}^{(\gamma, \delta)} \\
& ={ }_{(\gamma, \delta)} \quad\left(\chi_{Q_{1} I}^{(\gamma, \delta)}\right) \circ \chi_{Q_{2}}^{(\gamma, \delta)}={ }_{(\gamma, \delta)} \chi_{\left(Q_{1} I\right) Q_{2}}^{(\gamma, \delta)} .
\end{aligned}
$$

Thus $Q_{1} \cap I \cap Q_{2} \subseteq\left(Q_{1} I\right) Q_{2}$.

$($ iii $) \Rightarrow($ ii $)$ is obvious.

$(i i) \Rightarrow(i)$ Since $Q[a]=a \cup(S a \cap a S)$ and $I[a]=a \cup S a \cup a S$ are principle quasi-ideal and principle ideal of $S$ generated by a respectively. Thus by $(i i)$, 
left invertive law, medial law and Lemma 3 , we have,

$$
\begin{aligned}
& (a \cup(S a \cap a S)) \cap(a \cup S a \cup a S) \cap(a \cup(S a \cap a S)) \\
\subseteq & ((a \cup(S a \cap a S))(a \cup S a \cup a S))(a \cup(S a \cap a S)) \\
\subseteq & \{(a \cup a S) S\}(a \cap a S) \\
= & \{a S \cup(a S) S\}(a \cap a S) \\
= & (a S \cup S a)(a \cap a S) \\
= & \{(a S) a \cup(a S)(a S) \cup(S a) a \cup(S a) a S a \\
= & (a S) a \cup a^{2} S \cup a(a S) .
\end{aligned}
$$

Hence $S$ is regular.

Theorem 6. For an AG-groupoid $S$ with left identity, the following are equivalent:

(i) $S$ is regular.

(ii) For any principle bi-ideal $B[a], B[a]=(B[a] S) B[a]$.

(iii) For any bi-ideal $B, B=(B S) B$.

(iv) For any generalized bi-ideal $B, B=(B S) B$.

(v) For any $\left(\in_{\gamma}, \in_{\gamma} \vee q_{\delta}\right)$-fuzzy soft bi-ideal $\langle F, B\rangle$ over $S$, we have

$$
\langle F, B\rangle=_{(\gamma, \delta)}(\langle F, B\rangle \odot \Sigma(S, E)) \odot\langle F, B\rangle .
$$

(vi) For any $\left(\in_{\gamma}, \in_{\gamma} \vee q_{\delta}\right)$-fuzzy soft generalized bi-ideal $\langle F, B\rangle$ over $S$, we have

$$
\langle F, B\rangle=_{(\gamma, \delta)}(\langle F, B\rangle \odot \Sigma(S, E)) \odot\langle F, B\rangle .
$$

Proof. $(i) \Rightarrow(v i)$ Assume that $\langle F, B\rangle$ is $\left(\in_{\gamma}, \in_{\gamma} \vee q_{\delta}\right)$-fuzzy soft generalized biideal over a regular AG-groupoid $S$. Since $S$ is regular so for $b \in S$ there exist $x \in S$ such that $b=(b x) b$. First we have to show that $F(\epsilon) \subseteq \vee q_{(\gamma, \delta)}((F(\epsilon) \circ$ 
$\left.\chi_{S}^{\delta}\right) \circ F(\epsilon)$. Then we have

$$
\begin{aligned}
& \max \left\{\left(F(\epsilon) \circ \chi_{S}^{(\gamma, \delta)}\right) \circ F(\epsilon)(b), \gamma\right\} \\
= & \max \left\{\bigvee_{b=p q}\left\{\left(F(\epsilon) \circ \chi_{S}^{(\gamma, \delta)}\right)(p) \wedge F(\epsilon)(q)\right\}, \gamma\right\} \\
\geq & \max \left\{\left(F(\epsilon) \circ \chi_{S}^{(\gamma, \delta)}\right)(b x) \wedge F(\epsilon)(b), \gamma\right\} \\
= & \max \left\{\bigvee_{b x=c d}\left(F(\epsilon)(c) \wedge \chi_{S}^{(\gamma, \delta)}(d)\right) \wedge F(\epsilon)(b), \gamma\right\} \\
\geq & \max \left\{F(\epsilon)(b) \wedge \chi_{S}^{(\gamma, \delta)}(x) \wedge F(\epsilon)(b), \gamma\right\} \\
= & \min \{\max \{F(\epsilon)(b), \gamma\}, 1, \max \{F(\epsilon)(b), \gamma\}\} . \\
\geq & \min \{\min \{F(\epsilon)(b), \delta\}, \min \{F(\epsilon)(b), \delta\}\} \\
= & \min \{F(\epsilon)(b) \wedge F(\epsilon)(b), \delta\} \\
= & \min \{F(\epsilon)(b), \delta\}
\end{aligned}
$$

Thus $\min \{F(\epsilon)(b), \delta\} \leq \max \left\{\left(F(\epsilon) \circ \chi_{S}^{(\gamma, \delta)}\right) \circ F(\epsilon)(b), \gamma\right\}$. This implies that $F(\epsilon) \subseteq \vee q_{(\gamma, \delta)}\left(F(\epsilon) \circ \chi_{S}^{(\gamma, \delta)}\right) \circ F(\epsilon)$. That is $H(\epsilon) \subseteq \vee q_{(\gamma, \delta)}\left(F(\epsilon) \circ \chi_{S}^{(\gamma, \delta)}\right) \circ$ $F(\epsilon)$. Thus in any case, we have

$$
H(\epsilon) \subseteq \vee q_{(\gamma, \delta)}\left(F(\epsilon) \circ \chi_{S}^{(\gamma, \delta)}\right) \circ F(\epsilon) .
$$

Therefore,

$$
\left.\langle F, B\rangle \subset_{(\gamma, \delta)}(\langle F, B\rangle \odot \Sigma(S, E)) \odot F, B\right\rangle .
$$

Since $\langle F, B\rangle$ is any $\left(\in_{\gamma}, \in_{\gamma} \vee q_{\delta}\right)$-fuzzy soft generalized bi- ideal over $S$, we have

$$
(\langle F, B\rangle \odot \Sigma(S, E)) \odot F, B\rangle \subset_{(\gamma, \delta)}\langle F, B\rangle .
$$

Thus

$$
\left.\langle F, B\rangle=_{(\gamma, \delta)}(\langle F, B\rangle \odot \Sigma(S, E)) \odot F, B\right\rangle .
$$

$(v i) \Rightarrow(v)$ is obvious.

$(v) \Rightarrow(i v)$ Assume that $B$ is a bi-ideal of $S$. Assume that the given condition $(v)$ holds, then $\Sigma(B, E)$ be an $\left(\in_{\gamma}, \in_{\gamma} \vee q_{\delta}\right)$-fuzzy soft bi-ideal over $S$. Now by the assumption, we have

$$
\Sigma(B, E)=_{(\gamma, \delta)}(\Sigma(B, E) \odot \Sigma(S, E)) \odot \Sigma(B, E) .
$$

Hence we have

$$
\begin{aligned}
\chi_{B}^{(\gamma, \delta)} & ={ }_{(\gamma, \delta)} \quad\left(\chi_{B}^{(\gamma, \delta)} \circ \chi_{S}^{(\gamma, \delta)}\right) \circ \chi_{B}^{(\gamma, \delta)} \\
& ={ }_{(\gamma, \delta)} \quad\left(\chi_{B S}^{(\gamma, \delta)}\right) \circ \chi_{B}^{(\gamma, \delta)}={ }_{(\gamma, \delta)} \chi_{(B S) B}^{(\gamma, \delta)} .
\end{aligned}
$$


Therefore $B=(B S) B$.

$($ iv $) \Rightarrow($ iii $)$ is obvious.

$($ iii $) \Rightarrow($ ii $)$ is obvious.

(ii) $\Rightarrow(i)$

Since $B[a]=a \cup a^{2} \cup(a S) a$ is a principle bi-ideal of $S$ generated by $a$ respectively. Thus by (ii) and Lemma 3 , we have,

$$
\begin{aligned}
a \cup a^{2} \cup(a S) a= & {\left[\left\{a \cup a^{2} \cup(a S) a\right\} S\right]\left(a \cup a^{2} \cup(a S) a\right) } \\
= & {\left[a S \cup a^{2} S \cup\{(a S) a\} S\right]\left(a \cup a^{2} \cup(a S) a\right) } \\
= & \left(a S \cup a^{2} S \cup a(a S)\right)\left(a \cup a^{2} \cup(a S) a\right) \\
= & (a S) a \cup(a S) a^{2} \cup(a S)((a S) a) \\
& \cup\left(a^{2} S\right) a \cup\left(a^{2} S\right) a^{2} \cup\left(a^{2} S\right)((a S) a) \\
& \cup(a(a S)) a \cup(a(a S)) a^{2} \cup(a(a S))((a S) a) \\
= & (a S) a \cup a^{2} S \cup(a S) a \cup a^{2} S \cup a^{2} S \cup a^{2} S \\
& \cup(a S) a \cup(a S) a \cup(a S) a \\
= & a^{2} S \cup(a S) a .
\end{aligned}
$$

Hence $S$ is regular

Theorem 7. For an AG-groupoid $S$ with left identity, the following are equivalent:

(i) $S$ is regular.

(ii) $B[a] \cap Q[a] \subseteq(B[a] S) Q[a]$, for some $a$ in $S$.

(iii) For any bi-ideal $B$ and quasi-ideal $Q$ of $S, B \cap Q \subseteq(B S) Q$.

(iv) For any $\left(\in_{\gamma}, \in_{\gamma} \vee q_{\delta}\right)$-fuzzy soft bi-ideal $\langle F, B\rangle$ and $\left(\in_{\gamma}, \in_{\gamma} \vee q_{\delta}\right)$-fuzzy soft quasi-ideal $\langle G, Q\rangle$ over $S$, we have

$$
\langle F, B\rangle \cap_{\mathcal{E}}\langle G, Q\rangle \subset_{(\gamma, \delta)}(\langle F, B\rangle \odot \Sigma(S, E)) \odot\langle G, Q\rangle .
$$

(v) For any $\left(\in_{\gamma}, \in_{\gamma} \vee q_{\delta}\right)$-fuzzy soft generalized bi-ideal $\langle F, B\rangle$ and $\left(\in_{\gamma}, \in_{\gamma}\right.$ $\left.\vee q_{\delta}\right)$-fuzzy soft quasi-ideal $\langle G, Q\rangle$ over $S$, we have

$$
\langle F, B\rangle \cap_{\mathcal{E}}\langle G, Q\rangle \subset_{(\gamma, \delta)}(\langle F, B\rangle \odot \Sigma(S, E)) \odot\langle G, Q\rangle .
$$

Proof. $(i) \Rightarrow(v)$ Assume that $\langle F, B\rangle$ and $\langle G, Q\rangle$ are $\left(\epsilon_{\gamma}, \in_{\gamma} \vee q_{\delta}\right)$-fuzzy soft generalized bi-ideal and $\left(\epsilon_{\gamma}, \in_{\gamma} \vee q_{\delta}\right)$-fuzzy soft quasi-ideal over a regular AGgroupoid $S$, respectively. Let $a$ be any element of $S,\langle F, B\rangle \cap_{\mathcal{E}}\langle G, Q\rangle=$ $\left\langle K_{1}, B \cup Q\right\rangle$. For any $\epsilon \in B \cup Q$. We consider the following cases,

Case 1: $\epsilon \in B-Q$. Then $H(\epsilon)=F(\epsilon)=(F \circ G)(\epsilon)$.

Case 2: $\epsilon \in Q-B$. Then $H(\epsilon)=G(\epsilon)=(F \circ G)(\epsilon)$. 
Case 3: $\epsilon \in B \cap Q$. Then $H(\epsilon)=F(\epsilon) \cap G(\epsilon)$ and $(F \circ G)(\epsilon)=F(\epsilon) \circ G(\epsilon)$. First we show that $F(\epsilon) \cap G(\epsilon) \subseteq \vee q_{(\gamma, \delta)}\left(F(\epsilon) \circ \chi_{S}^{(\gamma, \delta)}\right) \circ G(\epsilon)$. Now since $S$ is regular, for $a \in S$ there exist $x \in S$ such that $a=(a x) a=[\{(a x) a\} x] a$ by left invertive law and the law $a(b c)=b(a c)$.

Thus we deduce that

$$
\begin{aligned}
& \max \left\{\left(F(\epsilon) \circ \chi_{S}^{(\gamma, \delta)}\right) \circ G(\epsilon)(a), \gamma\right\} \\
= & \max \left\{\bigvee_{a=p q}\left\{\left(F(\epsilon) \circ \chi_{S}^{(\gamma, \delta)}\right)(p) \wedge G(\epsilon)(q)\right\}, \gamma\right\} \\
\geq & \max \left\{\left(F(\epsilon) \circ \chi_{S}^{(\gamma, \delta)}\right)[\{(a x) a\} x] \wedge G(\epsilon)(a), \gamma\right\} \\
= & \max \left\{\bigvee_{[\{(a x) a\} x]=c d}\left(F(\epsilon)(c) \wedge \chi_{S}^{(\gamma, \delta)}(d)\right) \wedge G(\epsilon)(a), \gamma\right\} \\
\geq & \max \left\{F(\epsilon)\{(a x) a\} \wedge \chi_{S}^{(\gamma, \delta)}(x) \wedge G(\epsilon)(a), \gamma\right\} \\
= & \min \{\max \{F(\epsilon)\{(a x) a\}, \gamma\}, 1, \max \{G(\epsilon)(a), \gamma\}\} . \\
\geq & \min \{\min \{F(\epsilon)(a), \delta\}, \min \{G(\epsilon)(a), \delta\}\} \\
= & \min \{F(\epsilon)(a) \wedge G(\epsilon)(a), \delta\} \\
= & \min \{(F(\epsilon) \cap G(\epsilon))(a), \delta\}
\end{aligned}
$$

Hence $F(\epsilon) \cap G(\epsilon) \subseteq \vee q_{(\gamma, \delta)}\left(F(\epsilon) \circ \chi_{S}^{(\gamma, \delta)}\right) \circ G(\epsilon)$. That is,

$$
H(\epsilon) \subseteq \vee q_{(\gamma, \delta)}\left(F(\epsilon) \circ \chi_{S}^{(\gamma, \delta)}\right) \circ G(\epsilon) .
$$

Thus in any case, we have

$$
H(\epsilon) \subseteq \vee q_{(\gamma, \delta)}\left(F(\epsilon) \circ \chi_{S}^{(\gamma, \delta)}\right) \circ G(\epsilon) .
$$

Therefore,

$$
\langle F, B\rangle \cap_{\mathcal{E}}\langle G, Q\rangle \subset_{(\gamma, \delta)}(\langle F, B\rangle \odot \Sigma(S, E)) \odot\langle G, Q\rangle .
$$

$(v) \Rightarrow(i v)$ is obvious.

$($ iv $) \Rightarrow($ iii $)$

Assume that $B$ and $Q$ are bi-ideal and quasi-ideal of regular AG-groupioud of $S$ respectively, then $\Sigma(B, E)$ and $\Sigma(Q, E)$ be an $\left(\epsilon_{\gamma}, \epsilon_{\gamma} \vee q_{\delta}\right)$-fuzzy soft biideal and $\left(\epsilon_{\gamma}, \in_{\gamma} \vee q_{\delta}\right)$-fuzzy soft quasi- ideal $\langle G, Q\rangle$ over $S$. Now by the assumption, we have

$$
\Sigma(B, E) \cap_{\mathcal{E}} \Sigma(Q, E)=_{(\gamma, \delta)}(\Sigma(B, E) \odot \Sigma(S, E)) \odot \Sigma(Q, E) .
$$


So we have,

$$
\begin{aligned}
\chi_{(B \cap Q)}^{(\gamma, \delta)} & ={ }_{(\gamma, \delta)} \quad \chi_{B}^{(\gamma, \delta)} \cap \chi_{S}^{(\gamma, \delta)} \cap \chi_{Q}^{(\gamma, \delta)} \subseteq \vee q_{(\gamma, \delta)}\left(\chi_{B}^{(\gamma, \delta)} \circ \chi_{S}^{(\gamma, \delta)}\right) \circ \chi_{Q}^{(\gamma, \delta)} \\
& ={ }_{(\gamma, \delta)} \quad\left(\chi_{B S}^{(\gamma, \delta)}\right) \circ \chi_{Q}^{(\gamma, \delta)}={ }_{(\gamma, \delta)} \chi_{(B S) Q}^{(\gamma, \delta)} .
\end{aligned}
$$

Therefore $B \cap Q \subseteq(B S) Q$.

$($ iii $) \Rightarrow($ ii $)$ is obvious.

$(i i) \Rightarrow(i)$

Since $B[a]=a \cup a^{2} \cup(a S) a$ and $Q[a]=a \cup(S a \cap a S)$ are principle bi-ideal and principle quasi-ideal of $S$ generated by a respectively. Thus by (ii), Eq. (1), Lemma 3, medial law and left invertive law, we have

$$
\begin{aligned}
& \left\{a \cup a^{2} \cup(a S) a\right\} \cap\{a \cup(S a \cap a S)\} \\
\subseteq & \left\{\left(a \cup a^{2} \cup(a S) a\right) S\right\}(a \cup(S a \cap a S)) \\
\subseteq & \left\{a S \cup a^{2} S \cup((a S) a) S\right\}(a \cup S a) \\
= & \left\{a S \cup a^{2} S \cup(S a)(a S)\right\}(a \cup S a) \\
= & \left\{(a S) a \cup\left(a^{2} S\right) a \cup\{(S a)(a S)\} a \cup(a S)(S a)\right. \\
& \left.\cup\left(a^{2} S\right)(S a) \cup(S a)(a S)(S a)\right\} \\
\subseteq & (a S) a \cup S a^{2} \cup(a S) a \cup(a S) a \cup S a^{2} \cup(a S) a \\
= & (a S) a \cup a^{2} S
\end{aligned}
$$

Hence $S$ is regular as required.

\section{Conclusions}

We have applied the theory of fuzzy soft sets to algebraic ideal theory of regular AG-groupoids. We introduced $\left(\epsilon_{\gamma}, \in_{\gamma} \vee q_{\delta}\right)$-fuzzy soft left ideals (right ideals, bi-ideals and quasi-ideals) over AG-groupoids. Moreover, we presented some characterizations for regular AG-groupoids based on these newly introduced fuzzy soft ideals over them. The natural extension of this research work is to study intra-regular AG-groupoids using fuzzy soft ideals.

\section{Acknowledgments}

This work was partially supported by the National Natural Science Foundation of China (Program No. 11301415), Natural Science Basic Research Plan in Shaanxi Province of China (Program No. 2013JQ1020), Shaanxi Provincial Research Plan for Young Scientific and Technological New Stars (Program No. 2014KJXX-73), Scientific Research Program Funded by Shaanxi Provincial 
Education Department of China (Program No. 2013JK1098) and New Star Team of Xi'an University of Posts and Telecommunications. The Pakistani authors are thankful to the Higher Education Commission of Pakistan for financial support.

\section{References}

[1] L.A. Zadeh, Fuzzy sets, Information and Control 8 (1965) 338-353.

[2] Z. Pawlak, Rough sets, International Journal of Information and Computer Sciences 11 (1982) 341-356.

[3] M.B. Gorzalzany, A method of inference in approximate reasoning based on interval-valued fuzzy sets. Fuzzy Sets and Systems 21 (1987) 1-17.

[4] D. Molodtsov, Soft set theory-First results, Computer and Mathematics with Applications 37 (1999) 19-31.

[5] M. Akram, B. Davvaz, F. Feng, Intuitionistic Fuzzy Soft K-Algebras, Mathematics in Computer Science, 2013, 7 (3), 353-365.

[6] M. Akram, F. Feng, Soft intersection Lie algebras, Quasigroups and Related Systems, 2013, 21 (1), 11-18.

[7] M. Akram, N.O. Alshehri, R.S. Alghamdi, Fuzzy soft K-algebras, Utilitas Mathematica 90 (2013) 307-325.

[8] N.O. Alshehri, M. Akram, R.S. Al-ghamdi, Applications of Soft Sets in K-Algebras, Advances in Fuzzy Systems, Volume 2013 (2013), Article ID 319542, 8 pages, 2013.

[9] V. Leoreanu-Fotea, B. Davvaz, F. Feng, C. Chiper, Join spaces, soft join spaces and lattices, Analele Stiintifice Ale Universitatii Ovidius Constanta-Seria Matematica 22(1), 155-167, 2014.

[10] X. Liu, D. Xiang, J. Zhan, K.P. Shum, Isomorphism theorems for soft rings, Algebra Colloquium 19(4) (2012) 649-656.

[11] X. Liu, D. Xiang, J. Zhan, Fuzzy isomorphism theorems of soft rings, Neural Computing and Applications 21(2) (2012) 391-397.

[12] X. Ma, H.S. Kim, (M,N)-Soft Intersection BL-Algebras and Their Congruences, The Scientific World Journal, vol. 2014, Article ID 461060, 6 pages, 2014. 
[13] G. Muhiuddin, F. Feng, Y.B. Jun, Subalgebras of BCK/BCI-algebras based on cubic soft sets, The Scientific World Journal, vol. 2014, Article ID 458638, 9 pages.

[14] C.H. Park, Y.B. Jun, M.A. Öztürk, Soft WS-algebras, Communications of Korean Mathematical Society 23 (2008) 313-324.

[15] A. Sezgin, A.O. Atagun, E. Aygun, A note on soft near-rings and idealistic soft near-rings, Filomat 25 (2011) 53-68.

[16] A. Sezgin, A.O. Atagun, Soft groups and normalistic soft groups, Comput. Math. Appl. 62 (2011) 685-698.

[17] S.Z. Song, H.S. Kim, Y.B. Jun, Ideal Theory in Semigroups Based on Intersectional Soft Sets, The Scientific World Journal, vol. 2014, Article ID 136424, 7 pages, 2014.

[18] D.R.P. Williams, A.B. Saeid, Fuzzy soft ideals in subtraction algebras, Neural Computing and Applications 21 (2012) 159-169.

[19] X. Xin and W. Li, Soft Congruence Relations over Rings, The Scientific World Journal, vol. 2014, Article ID 541630, 9 pages, 2014.

[20] Y. Yang, X. Xin, P. He, Applications of Soft Union Sets in the Ring Theory, Journal of Applied Mathematics, vol. 2013, Article ID 474890, 9 pages, 2013.

[21] J. Zhan, Y.B. Jun, Soft BL-algebras based on fuzzy sets, Computers and Mathematics with Applications 59 (2010) 2037-2046.

[22] P. V. Protić and N. Stevanović, On Abel-Grassmann's groupoids, Proc. Math. Conf. Priština, (1994), 31-38.

[23] Y.Q. Yin and J. Zhan, Characterization of ordered semigroups in terms of fuzzy soft ideals, Bull. Malays. Math. Sci. Soc. 35 (2012), 997-1015.

[24] P.K. Maji, R. Biswas, A.R. Roy, Fuzzy soft sets, J. Fuzzy Math. 9(3) (2001) 589-602.

[25] M.I. Ali, F. Feng, X. Liu, W.K. Min, M. Shabir, On some new operations in soft set theory, Comput. Math. Appl. 57 (2009) 1547-1553.

Feng FENG,

Department of Applied Mathematics, School of Science,

Xi'an University of Posts and Telecommunications,

Xi'an 710121, China.

Email: fengnix@hotmail.com 
Madad KHAN,

Department of Mathematics,

COMSATS Institute of Information Technology,

Abbottabad 22060, Pakistan.

Email: madadmath@yahoo.com

Violeta LEOREANU-FOTEA,

Faculty of Mathematics,

"Alexandru Ioan Cuza" University,

700506 Iaşi, Romania.

Email: leoreanu2002@yahoo.com

Saima ANIS,

Department of Mathematics,

COMSATS Institute of Information Technology,

Abbottabad 22060, Pakistan.

Email: saimaanispk@gmail.com

Nadeem AJAIB,

Department of Mathematics,

COMSATS Institute of Information Technology,

Abbottabad 22060, Pakistan.

Email: nadeem.ajaib@yahoo.com 